\title{
Modified Overall Immune Related Response Criterion, Stable Disease
}

National Cancer Institute

\section{Source}

National Cancer Institute. Modified Overall Immune Related Response Criterion, Stable

Disease. NCI Thesaurus. Code C111086.

The evaluation of the antitumor response shows that the sum of the longest diameters of target and new measurable lesions do not meet the criteria for complete remission, partial response (compared to baseline) or progressive disease (compared to nadir). 